\title{
Spitzer steps aside
}

\author{
The Spitzer Space Telescope recently ceased operations, powering down its remaining detector after more than a \\ decade and a half of revealing the infrared Universe. Its legacy will be continued by far more expensive missions \\ that will have big boots to fill.
}

$\mathrm{T}$ he Spitzer Space Telescope was launched in 2003 and continued operating until it was put into safe mode by NASA indefinitely earlier this year. It followed in a great line of predecessors: the Infrared Astronomical Satellite (1983) and the Infrared Space Observatory (19951998), both helium-cooled telescopes with $60 \mathrm{~cm}$ diameter mirrors. Spitzer - also helium-cooled for the initial six-year period, but with a larger, $85 \mathrm{~cm}$ diameter mirror - was the mid-infrared representative in NASA's Great Observatories programme. The Spitzer mission could have continued slightly longer than its 16 years, but in NASA senior review it was decided to retire the mission now to cede resources to what is to be the next great infrared observatory, the James Webb Space Telescope (JWST), due to launch next year.

In celebration of the Spitzer mission we dedicate this issue of Nature Astronomy - and the next - to exploring what Spitzer contributed to our understanding of the infrared Universe. Spitzer leaves a substantial scientific legacy across many fields of astronomy and planetary science that is spread throughout more than 8,000 peer-reviewed papers. It would be impossible to make a complete summary of everything that Spitzer has enabled us to learn about the Universe, but in this retrospective we are publishing a series of broad Review Articles covering certain topics, alongside some shorter articles from the architects and users of the Spitzer Space Telescope. All Spitzer-related articles will be gathered together in an online collection.

In this April issue, we start with three Review Articles covering subjects near and far. Christine Chen, Kate Su and Siyi Xu summarize Spitzer's contribution to the study of debris disks around main sequence and white dwarf stars. Spitzer seems built for studying these dusty remnants of planetary systems that emit light across the infrared spectrum, but interestingly only a small number were known before Spitzer came along, and only the closest had been well characterized. Looking at larger scales, Aigen Li reviews the polycyclic aromatic hydrocarbon (PAH) molecules that emit in distinctive bands across the wavelength range of Spitzer's Infrared Array Camera and Infrared Spectrograph instruments. PAHs are excellent tracers of conditions in astrophysical regions from planet-forming disks through to the interstellar media of high-redshift galaxies. The final Review in this issue comes from Mark Lacy and Anna Sajina, who select some of the highlights of Spitzer studies of active galactic nuclei (AGNs). Spitzer was able to identify a population of highly obscured AGNs, and then separate the different dust components of these distant galaxies. Forthcoming Review Articles in the series will cover exoplanets and brown dwarfs, the inner and outer Solar System, (ultra-)luminous infrared galaxies and high-redshift galaxies.

Spitzer project scientist Michael Werner shares his unique view of the mission as it has developed, matured, bore fruit and concluded over more than 40 years. In a candid and touching interview, he takes us through the different manifestations of the Spitzer concept before it launched, the nail-biting moments while in development, and the lessons that have come from leading such a successful and long-lived mission. Jan Cami, on the other hand, provides his perspective as a user of Spitzer. His team discovered a new extraterrestrial allotrope of carbon by looking at a Spitzer Infrared Spectrograph spectrum of a planetary nebula, realizing a decade-long personal quest. He talks through the lead-up to the discovery of cosmic buckyballs, the public and academic response and the sprouting of a new branch of astrochemistry. Spitzer facility scientist Thomas Roellig rounds up the Spitzer content of this issue with an overview of the instrumental payload of the telescope in our Mission Control.

While much of this issue is a retrospective, a component of many of the Review Articles is forward-looking. It is clear that Spitzer answered many scientific questions - some of which we did not even know to ask at the time of launch - but also that it asks further questions that we still need to find the answers to. The gauntlet will be picked up by the next generation of infrared space telescopes: SPHEREx, SPICA, WFIRST and, particularly, JWST. JWST will reinstate our ability to take mid-infrared spectra from space, which ceased when Spitzer exhausted its helium coolant supply.
JWST, with its greater angular resolution $\left(<0.5^{\prime \prime}\right)$ and increased sensitivity over a similar wavelength range and field of view, is a natural successor to Spitzer, and indeed many of the science cases for JWST are built on the previous progress brought about by Spitzer and its contemporaries (Herschel, WISE, Akari). JWST spectroscopy will certainly bring further insight into the atmospheres of the unique TRAPPIST-1 exoplanet system that Spitzer was pivotal in characterizing, and at the other end of the distance ladder will provide detailed physical and chemical information on galaxies close to the era of reionization that Spitzer revealed to us. Spitzer's science legacy will provide a foundation for years to come, particularly since the treasure trove of data in the Infrared Science Archive has only been partially explored.

On an equal footing with the science, the other outstanding legacy that Spitzer has left us with is in space telescope operations. As pointed out in an upcoming Review, operating the Spitzer Space Telescope led to significant developments in how to plan and carry out long-duration observations with minimal spacecraft disturbances and the mitigation of pointing fluctuations. Spitzer pioneered the Earth-trailing orbit, facilitating the operation of the subsequent Kepler Space Observatory, also from an Earth-trailing orbit. The Spitzer instruments were carefully characterized both before and after launch, making data reduction more straightforward. Specialist tools were created to reduce and analyse Spitzer data and novel techniques were implemented to remove instrumental noise, often with much community involvement. So while Spitzer will be quite rightly remembered for its monumental scientific discoveries (many of which are revisited in these pages) and beautiful images of optically obscured regions (see our Picture Story), future users and operators of the comparatively opulent and more expansive JWST and WFIRST will owe a huge debt of gratitude to the humbly persistent telescope with a mirror no larger than a hula hoop.

Published online: 14 April 2020 https://doi.org/10.1038/s41550-020-1089-0 